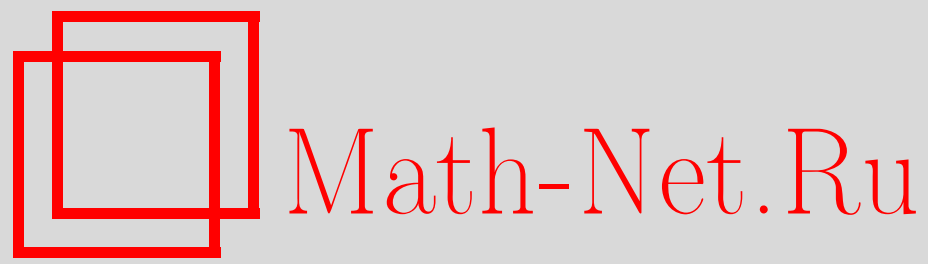

А. И. Буфетов, Операторные эргодические теоремы для действий свободных полугрупп и групп, Функи. анализ и его прил., 2000, том 34, выпуск 4, 1-17

DOI: https://doi.org/10.4213/faa322

Использование Общероссийского математического портала MathNet.Ru подразумевает, что вы прочитали и согласны с пользовательским соглашением

http://www . mathnet.ru/rus/agreement

Параметры загрузки:

IP : 3.80 .253 .173

26 апреля 2023 г., 15:09:22

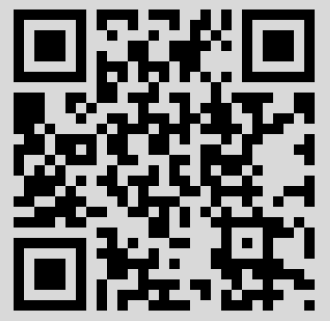




\title{
Операторные эргодические теоремы для действий свободных полугрупп и групп*
}

\author{
(C) 2000. А. И. БУФЕТОВ
}

\section{§1. Введение}

В работе получены новые эргодические теоремы для действий свободных по лугрупп и групп сохраняющими меру отображениями. Эти теоремы выводятся и классических эргодических теорем для марковских операторов.

Первые эргодические теоремы для действий произвольных счетных групп был получены в 1965 г. Оселедцем [2], следовавшим идее Какутани [3]. Для действи свободной группы в 1968 г. Гиварш [19] исследовал чезаровские средние равно мерных средних по сферам возрастающего радиуса в группе и получил для ни статистическую эргодическую теорему. В 1986 г. Григорчук [7] анонсировал ин дивидуальную эргодическую теорему для этих средних. В 1994 г. Нево [16] Нево и Стайн [18] опубликовали доказательство индивидуальной эргодическо теоремы. В 1999 г. Григорчук [8] анонсировал также эргодическую теорему дл действий свободных полугрупп.

В настоящей работе предлагается новый способ составления временны́х сред них. Пусть свободная полугруппа или группа действует на вероятностном про странстве сохраняющими меру отображениями. Зафиксируем набор образующи в нашей полугруппе и рассмотрим произвольную стационарную марковскую мер $\mu$ на пространстве бесконечных последовательностей образующих. Рассмотри сферу в нашей полугруппе и усредним ее элементы с весами, соответствующим мере $\mu$. Пусть $c_{n}^{\mu}-$ чезаровские средние сферических средних. Теорема 1 ест индивидуальная эргодическая теорема для средних $c_{n}^{\mu}$.

Основная идея доказательства - поставить в соответствие полугрупповом действию и мере $\mu$ специально построенный марковский оператор $T_{\mu}$. Класси ческие эргодические теоремы для оператора $T_{\mu}$ дают эргодические теоремы дл средних $c_{n}^{\mu}$. Этот подход позволяет получить операторные эргодические теорем для действий свободных полугрупп и групп (теорема 2).

Теорема 2 позволяет получить эргодические теоремы для действий полугрупг названных в работе строго марковскими. Неформально говоря, полугруппа ил группа называется строго марковской, если множество ее элементов находитс во взаимно однозначном соответствии с множеством допустимых слов неприво димой цепи Маркова с конечным числом состояний (точное определение строг марковских полугрупп и эргодическая теорема для них сформулированы в §9).

Работа построена следующим образом. В $\$ 2$ описан процесс усреднения, сфор мулирована эргодическая теорема (теорема 1) и введен марковский оператор $T_{\mu}$ 
играющий главную роль во всех доказательствах. В \$3 сформулирована теоре ма 2, операторный вариант теоремы 1 . Теорема 2 доказана в $\$ \$ 4,5$; из нее следуе теорема 1 . В $\$ 6$ обсуждаются необходимые условия инвариантности предела в те оремах 1,2 . В $\$ 7$ поточечная сходимость средних $c_{n}^{\mu}$ доказана для произвольно вероятностной инвариантной относительно сдвига меры $\mu$ на пространстве после довательностей из образующих. Ничего, однако, не говорится об инвариантност предела в этом общем случае. В $\$ 8$ инвариантность предела установлена и эрго дические теоремы доказаны в случае, когда $\mu$ - марковская мера порядка $k$. В $\S$ получены эргодические теоремы для действий строго марковских полугрупп.

Я глубоко благодарен Д. В. Аносову, А. М. Вершику, М. Л. Громову, Б. М. Гу ревичу, Ю. С. Ильяшенко, В. А. Каймановичу, М. Лину, А. Нево, В. И. Оселедцу Я. Г. Синаю, В. А. Тиморину, Ж.-П. Тувено, Дж. Фельдману и А. Ю. Фишкин за полезные обсуждения.

Я особенно признателен Р. И. Григорчуку. Его результаты и общение с ни оказали на меня сильное влияние. Эта работа - продолжение начатых им иссле дований.

\section{§2. Формулировка эргодической теоремы и набросок доказательства}

В этом параграфе сформулирован основной результат работы и дан набросо его доказательства.

Пусть $(X, \nu)$ - вероятностное пространство, $f_{1}, \ldots, f_{m}: X \rightarrow X-$ измеримы отображения, сохраняющие меру $\nu$, и $T_{1}, \ldots, T_{m}: L_{1}(X, \nu) \rightarrow L_{1}(X, \nu)-$ соот ветствующие этим отображениям операторы (т. е. $T_{i} \varphi=\varphi \circ f_{i}$ для $\varphi \in L_{1}(X, \nu)$ )

Рассмотрим пространство $\Omega_{m}$ односторонних бесконечных последовательно стей из символов $1, \ldots, m$ :

$$
\Omega_{m}=\left\{\omega=\omega_{1} \omega_{2} \ldots \omega_{n} \ldots: \omega_{i}=1, \ldots, m\right\} .
$$

Пусть $\sigma_{m}-$ сдвиг на $\Omega_{m}$, определенный формулой $\left(\sigma_{m} \omega\right)_{i}=\omega_{i+1}$.

Пусть $W_{m}$ есть множество всех конечных слов из символов $1, \ldots, m$ :

$$
W_{m}=\left\{w=w_{1} w_{2} \ldots w_{n}: w_{i}=1, \ldots, m\right\} .
$$

Через $|w|$ обозначим длину слова $w$. Для всякого $w \in W_{m}$ рассмотрим мно жество $C(w) \subset \Omega_{m}$ всех последовательностей, начинающихся словом $w$. Дл произвольной борелевской меры $\mu$ на $\Omega_{m}$ положим $\mu(w)=\mu(C(w))$.

Для всякого $w \in W_{m}, w=w_{1} \ldots w_{n}$, определим оператор

$$
T_{w}=T_{w_{n}} T_{w_{n-1}} \ldots T_{w_{1}} .
$$

Пусть $\mu$ есть $\sigma_{m}$-инвариантная борелевская вероятностная мера на $\Omega_{m}$. За фиксируем $|w|=l$ и рассмотрим сумму операторов $T_{w}$ с весами $\mu(w)$ :

$$
s_{l}^{\mu}(T)=\sum_{|w|=l} \mu(w) T_{w} .
$$

Усредним $s_{l}^{\mu}(T)$ по $l=0, \ldots, n-1$ :

$$
c_{n}^{\mu}(T)=\frac{1}{n} \sum_{l=0}^{n-1} s_{l}^{\mu}(T) .
$$


Пусть $\mu$ есть $\sigma_{m}$-инвариантная марковская мера на $\Omega_{m}$. Тогда, как мы дока жем, средние $c_{n}^{\mu}(T) \varphi$ сходятся почти всюду и в $L_{1}(X, \nu)$ для всякой суммируемо функции $\varphi$. Для описания их предела нужны дополнительные предположения.

ОПрЕДЕЛЕНИЕ 1. Матрицу $Q$ с неотрицательными элементами будем называт неприводимой, если при некотором $n>0$ все элементы матрицы $Q+Q^{2}$ $\cdots+Q^{n}$ положительны. (Если $Q$ стохастическая, это условие означает, что соответствующей цепи Маркова любое состояние достижимо из любого другого)

ОПреДЕЛЕНИЕ 2. Матрицу $P$ с неотрицательными элементами назовем $c$ ильн неприводимой, если $P$ неприводима и $P P^{T}$ неприводима (здесь $P^{T}-$ транспо нированная матрица $P$.)

ОПРЕДЕЛЕНИЕ 3. Марковскую цепь будем называть сильно неприводимой, ес ли ее матрица переходных вероятностей сильно неприводима. Марковскую мер будем называть сильно неприводимой, если отвечающая ей цепь сильно непри водима.

Теорема 1. Пусть $(X, \nu)$ - вероятностное пространство, $f_{1}, \ldots, f_{m}$ $X \rightarrow X-$ отображения, сохраняющие меру $\nu, u T_{1}, \ldots, T_{m}: L_{1}(X, \nu) \rightarrow L_{1}(X, \nu$ - соответствующие этим отображениям операторы.

Пусть н есть $\sigma_{m}$-инвариантная марковская мера на $\Omega_{m}$.

Тогда для всякой функизии $\varphi \in L_{1}(X, \nu)$ найдется такая функция $\bar{\varphi} \in L_{1}(X, \nu)$ что $c_{n}^{\mu}(T) \varphi \rightarrow \bar{\varphi}$ почти всюду по мере $\nu$ и в $L_{1}(X, \nu)$ при $n \rightarrow \infty$, приче $\int_{X} \varphi d \nu=\int_{X} \bar{\varphi} d \nu$.

Если мера $\mu$ сильно неприводима, то $T_{j} \bar{\varphi}=\bar{\varphi}, j=1, \ldots, m$.

Если $\varphi \in L_{p}(X, \nu), p \geqslant 1, \operatorname{mo~}_{n}^{\mu}(T) \varphi \rightarrow \bar{\varphi}$ также и в $L_{p}(X, \nu)$.

Пусть $I_{X}$ есть $\sigma$-алгебра $f_{1}, \ldots, f_{m}$-инвариантных подмножеств пространств $X$. Из теоремы 1 следует, что если мера $\mu$ сильно неприводима, то функция есть условное математическое ожидание функции $\varphi$ по отношению к $I_{X}$. Пуст $\left(p_{1}, \ldots, p_{n}\right)$ - начальное распределение, а $\left(p_{i j}\right)$ - переходные вероятности ме ры $\mu$.

Основная идея доказательства теоремы 1 состоит в том, что мере $\mu$ и опє раторам $T_{1}, \ldots, T_{m}$ ставится в соответствие марковский оператор $T_{\mu}: L_{1}(X, \nu)^{r}$ $\rightarrow L_{1}(X, \nu)^{m}$, определенный формулой

$$
T_{\mu}\left(\varphi_{1}, \ldots, \varphi_{m}\right)=\left(\psi_{1}, \ldots, \psi_{m}\right), \quad \text { где } \psi_{j}=\sum_{i=1}^{m} \frac{p_{i} p_{i j}}{p_{j}} T_{j} \varphi_{i} .
$$

Отождествим пространство $L_{1}(X, \nu)^{m}$ с пространством $L_{1}(X \times\{1, \ldots, m\}$ $\nu \times p)$, где мера $\nu \times p$ есть произведение меры $\nu$ на $X$ и распределения ве роятностей $p=\left(p_{1}, \ldots, p_{m}\right)$ на $\{1, \ldots, m\}$. При этом отождествлении опера тор $T_{\mu}$ становится сохраняющим меру марковским оператором на пространств $L_{1}(X \times\{1, \ldots, m\}, \nu \times p)$. Из эргодической теоремы для оператора $T_{\mu}$ следуе теорема 1 ; детали рассуждения - в $\$ 4,5$.

Теорема 1 обобщает эргодические теоремы Григорчука $[7,8]$, Нево [16] и Нево Стайна [18] для сохраняющих меру действий свободных полугрупп и групп.

Чтобы получить эргодические теоремы из $[7,18]$ для действий свободной груп пы с $l$ образующими, положим $m=2 l$, обозначим образующие через $g_{1}, \ldots, g l$ положим $g_{l+i}=g_{i}^{-1}$ и определим марковскую цепь таким образом: положи $p_{i, i+l}=p_{i+l, i}=0$ при $l \geqslant 1$ и $p_{i j}=1 /(m-1)$ при $|i-j| \neq l$ (т.е. пере ходы из образующего в его обратный и наоборот запрещены, а все остальны 
переходы равновероятны). Стационарное распределение этой цепи есть, очевид но, $(1 / m, \ldots, 1 / m)$; пусть $\mu-$ соответствующая стационарная марковская мер на $\Omega_{m}$. Мера $\mu$ сильно неприводима, а средние $s_{l}^{\mu}(T)$ суть равномерные средни по сферам в свободной группе; эргодическая теорема для их чезаровских средни следует теперь из теоремы 1.

\section{§3. Операторная эргодическая теорема}

В этом параграфе сформулирован операторный вариант теоремы 1 .

Пусть $(X, \nu)$ - пространство Лебега, и пусть $T_{1}, \ldots, T_{m}: L_{1}(X, \nu) \rightarrow L_{1}(X, \nu$ - линейные операторы. Операторы $T_{w}, s_{l}^{\mu}(T)$ и $c_{n}^{\mu}(T)$ вводятся по формула (1) $-(3)$.

Напомним стандартную терминологию.

Линейный оператор на банаховом пространстве называется сжатием, если ег норма не превосходит единицы.

Линейный оператор $T: L_{1}(X, \nu) \rightarrow L_{1}(X, \nu)$ называется положительным, ес ли образ неотрицательной функции под его действием есть неотрицательна функция.

Линейный оператор $T: L_{1}(X, \nu) \rightarrow L_{1}(X, \nu)$ называется $L_{1}-L_{\infty}$-сжатием, ес ли $\|T\|_{L_{1}} \leqslant 1,\|T\|_{L_{\infty}} \leqslant 1$.

Линейный оператор $T: L_{1}(X, \nu) \rightarrow L_{1}(X, \nu)$ coхраняет меру $\nu$, если для все $\varphi \in L_{1}(X, \nu)$ имеем

$$
\int_{X} \varphi d \nu=\int_{X} T \varphi d \nu .
$$

ТеОРема 2. Пусть н есть $\sigma_{m}$-инвариантная марковская мера на $\Omega_{m}$.

Пусть $\nu(X)<\infty$ и $T_{1}, \ldots, T_{m}-$ положительные $L_{1}-L_{\infty}$-сжатия. Тогда дл всякой функции $\varphi \in L_{1}(X, \nu)$ найдется функция $\bar{\varphi} \in L_{1}(X, \nu)$, такая, чт $c_{n}^{\mu}(T) \varphi \rightarrow \bar{\varphi}$ почти всюду по мере $\nu$ и в $L_{1}(X, \nu)$ nри $n \rightarrow \infty$.

Если мера н сильно неприводима, то $T_{i} \bar{\varphi}=\bar{\varphi}, i=1, \ldots, m$.

Если операторь $T_{1}, \ldots, T_{m}$ сохраняют меру $\nu$, mо $\int_{X} \varphi d \nu=\int_{X} \bar{\varphi} d \nu$. Eсл $p \geqslant 1, \operatorname{mo~} c_{n}^{\mu}(T) \varphi \rightarrow \bar{\varphi}$ также и в $L_{p}(X, \nu)$.

Теорема 1 вытекает из теоремы 2.

Теперь мы формулируем две леммы, одна из которых есть операторная эрго дическая теорема в гильбертовом пространстве, а другая - в $L_{p}, p>1$.

ЛЕмма 1. Пусть н есть $\sigma_{m}$-инвариантная марковская мера на $\Omega_{m}$.

Пусть $H$ - гильбертово пространство, и пусть линейные оператор $T_{1}, \ldots, T_{m}: H \rightarrow H-$ сжатия. Тогда для всякого $h \in H$ найдется векто $\bar{h} \in H$, такой, что $c_{n}^{\mu}(T) h \rightarrow \bar{h}$ в $H$ при $n \rightarrow \infty$.

Если мера н сильно неприводима, то $T_{i} \bar{h}=\bar{h}, i=1, \ldots, m$.

ЛЕмма 2. Пусть н есть $\sigma_{m}$-инвариантная марковская мера на $\Omega_{m},(X, \nu$ - пространство Лебега и $p>1$.

Пусть $T_{1}, \ldots, T_{m}: L_{p}(X, \nu) \rightarrow L_{p}(X, \nu)-$ положительнье сжатия. Тогд для всякой функции $\varphi \in L_{p}(X, \nu)$ найдется функция $\bar{\varphi} \in L_{p}(X, \nu)$, такая, ит $c_{n}^{\mu}(T) \varphi \rightarrow \bar{\varphi}$ почти всюду по мере $\nu$ и в $L_{p}(X, \nu)$ nри $n \rightarrow \infty$.

Если мера н сильно неприводима, то $T_{i} \bar{\varphi}=\bar{\varphi}, i=1, \ldots, m$.

Леммы 1, 2 и теорема 2 доказаны в $\$ \$ 4,5$. 


\section{§4. Сходимость временны́х средних}

В этом параграфе доказана сходимость временны́х средних в теоремах 1 и 2 Основная идея - оператор $T_{\mu}$, вводимый так же, как в $§ 2$.

Пусть $L-$ линейное пространство над $\mathbb{R}$ или $\mathbb{C}, T_{1}, \ldots, T_{m}: L \rightarrow L-$ линей ные операторы и $\mu$ есть $\sigma_{m}$-инвариантная марковская мера на $\Omega_{m}$ с начальны распределением $\left(p_{1}, \ldots, p_{m}\right)$ и матрицей переходных вероятностей $P=\left(p_{i j}\right) . M_{\mathrm{l}}$ всегда будем считать, что $p_{i}>0$ при всех $i=1, \ldots, m$.

Рассмотрим взвешенную сумму операторов $T_{w}$ по словам длины $l$ с фиксиро ванным последним символом:

$$
s_{l}^{\mu, i}(T)=\sum_{\left\{w:|w|=l, w_{l}=i\right\}} \mu(w) T_{w} .
$$

Усредним $s_{l}^{\mu, i}(T)$ по $l=0, \ldots, n-1$ :

$$
c_{n}^{\mu, i}(T)=\frac{1}{n} \sum_{l=0}^{n-1} s_{l}^{\mu, i}(T) .
$$

ЛЕмма 3. Для любого натурального $l$ и ґюбого $j \in\{1, \ldots, m\}$ выполняетс равенство

$$
s_{l+1}^{\mu, j}=\sum_{i=1}^{m} p_{i j} T_{j} s_{l}^{\mu, i} .
$$

Доказательство - прямое вычисление.

Мы можем переписать (7) так:

$$
\frac{s_{l+1}^{\mu, j}}{p_{j}}=\sum_{i=1}^{m} \frac{p_{i} p_{i j}}{p_{j}} T_{j}\left(\frac{s_{l}^{\mu, i}}{p_{i}}\right) .
$$

Теперь рассмотрим пространство $L^{m}, m$-ю декартову степень пространства $L$ Операторам $T_{1}, \ldots, T_{m}$ и мере $\mu$ поставим в соответствие оператор $T_{\mu}: L^{m} \rightarrow L^{m}$ определенный формулой

$$
T_{\mu}\left(v_{1}, \ldots, v_{m}\right)=\left(\tilde{v}_{1}, \ldots, \tilde{v}_{m}\right), \quad \text { где } \tilde{v}_{j}=\sum_{i=1}^{m} \frac{p_{i} p_{i j}}{p_{j}} T_{j} v_{i} .
$$

ЛЕмма 4. Для любого $v \in L$ и ююбого $n \in \mathbb{N}$ имеем

$$
T_{\mu}^{n}(v, \ldots, v)=\left(\frac{s_{l}^{\mu, 1}(T) v}{p_{1}}, \ldots, \frac{s_{l}^{\mu, m}(T) v}{p_{m}}\right) .
$$

Это следует из (7) и (8).

СлЕДСТВИЕ 1. Для любого $v \in L$ и любого $n \in \mathbb{N}$ имеем

$$
\frac{1}{n} \sum_{l=0}^{n-1} T_{\mu}^{n}(v, \ldots, v)=\left(\frac{c_{n}^{\mu, 1}(T) v}{p_{1}}, \ldots, \frac{c_{n}^{\mu, m}(T) v}{p_{m}}\right) .
$$

Это вытекает из леммы 4.

Применяя классические операторные эргодические теоремы к оператору $T_{\mu}$ используя следствие 1 , мы получаем утверждения о сходимости средних $c_{n}^{\mu}(T)$. 
ЛЕмма 5. Пусть $H-$ гильбертово пространство, пусть $T_{1}, \ldots, T_{m}: H \rightarrow I$ - сжатия, и пусть н есть $\sigma_{m}$-инвариантная марковская мера на $\Omega_{m}$. Тогд для любого $h \in H$ и любого $i \in\{1, \ldots, m\}$ последовательность $\left(1 / p_{i}\right) c_{n}^{\mu, i}(T)$ сходится в $H$ при $n \rightarrow \infty \kappa$ некоторому вектору $\bar{h}_{i}$, причем $T_{\mu}\left(\bar{h}_{1}, \ldots, \bar{h}_{m}\right)$ $\left(\bar{h}_{1}, \ldots, \bar{h}_{m}\right)$.

ДокАЗАТЕЛЬСтво. Если $T_{1}, \ldots, T_{m}-$ сжатия в $H$, то $T_{\mu}-$ сжатие в $H^{m}$ Следствие 1 и эргодическая теорема для $T_{\mu}$ завершают доказательство.

Равенство $c_{n}^{\mu}(T)=c_{n}^{\mu, 1}(T)+\cdots+c_{n}^{\mu, m}(T)$ дает

СледствиЕ 2. В предположениях леммы 5 для всякого $h \in H$ последователь ность $c_{n}^{\mu}(T) h$ сходится в $H$.

Следствие 2 доказывает сходимость временны́х средних в лемме 1 .

Аналогичные утверждения имеют место в произвольных рефлексивных бана ховых пространствах.

ЛЕмма 6. Пусть $L$ - рефлексивное банахово пространство, пуст $T_{1}, \ldots, T_{m}: L \rightarrow L-$ сжатия, и пусть н есть $\sigma_{m}$-инвариантная марковска мера на $\Omega_{m}$. Тогда для всякого $v \in L$ и всякого $i \in\{1, \ldots, m\}$ последова тельность $\left(1 / p_{i}\right) c_{n}^{\mu, i}(T) v$ сходится в $L$ при $n \rightarrow \infty \kappa$ некоторому $\bar{v}_{i}$, приче $T_{\mu}\left(\bar{v}_{1}, \ldots, \bar{v}_{m}\right)=\left(\bar{v}_{1}, \ldots, \bar{v}_{m}\right)$.

ДокАЗАТЕЛЬство. Если $T_{1}, \ldots, T_{m}-$ сжатия в $L$, то $T_{\mu}-$ сжатие в $L^{m}$ Следствие 1 и эргодическая теорема Лорча для сжатия $T_{\mu}$ (см. [20] или [2] р. 73]) завершают доказательство.

СледствиЕ 3. В предположениях леммы 6 для всякого $v \in L$ последователь ность $c_{n}^{\mu}(T) v$ сходится в L.

Теперь пусть $(X, \nu)$ - пространство Лебега, а $T_{1}, \ldots, T_{m}: L_{1}(X, \nu) \rightarrow L_{1}(X, \nu$ - линейные операторы.

Пусть $\mu$ есть $\sigma_{m}$-инвариантная марковская мера на $\Omega_{m}$ с начальным распре делением $p=\left(p_{1}, \ldots, p_{m}\right)$ и матрицей переходных вероятностей $P=\left(p_{i j}\right)$. Пуст $T_{\mu}: L_{1}(X, \nu)^{m} \rightarrow L_{1}(X, \nu)^{m}$ - оператор, определенный формулой $(9)$.

Пространство $L_{1}(X, \nu)^{m}$ можно отождествить с пространством $L_{1}(X$ $\{1, \ldots, m\}, \nu \times p)$, где мера $\nu \times p$ есть произведение меры $\nu$ на $X$ и распределе ния вероятностей $p=\left(p_{1}, \ldots, p_{m}\right)$ на $\{1, \ldots, m\}$. Оператор $T_{\mu}$ теперь становитс оператором на пространстве $L_{1}(X \times\{1, \ldots, m\}, \nu \times p)$. Ясно, что если $T_{1}, \ldots, T_{r}$ положительны, то положителен и $T_{\mu}$, если $T_{1}, \ldots, T_{m}$ суть $L_{1}$-сжатия, то и $T$ является $L_{1}$-сжатием, если $T_{1}, \ldots, T_{m}$ суть $L_{\infty}$-сжатия, то и $T_{\mu}$ есть $L_{\infty}$-сжатиє а если $T_{1}, \ldots, T_{m}$ сохраняют меру $\nu$, то $T_{\mu}$ сохраняет меру $\nu \times p$.

ЛЕмма 7. Пусть $(X, \nu)$ - вероятностное пространство, и пуст $T_{1}, \ldots, T_{m}-$ положительные $L_{1}-L_{\infty}$-сжатия. Тогда для любой функции $\varphi$ $L_{1}(X, \nu)$ и любого $i=1, \ldots, m$ последовательность $c_{n}^{\mu, i}(T) \varphi$ сходится $\nu$-почт всюду и в $L_{1}(X, \nu)$ рри $n \rightarrow \infty$. Если $\bar{\varphi}_{i}=\lim _{n \rightarrow \infty}\left(1 / p_{i}\right) c_{n}^{\mu, i}(T) \varphi$, mо $T_{\mu}\left(\bar{\varphi}_{1}, \ldots, \bar{\varphi}_{m}\right.$ $=\left(\bar{\varphi}_{1}, \ldots, \bar{\varphi}_{m}\right)$.

Напомним стандартный факт (см. $[12,21])$.

Теорема 3. Если $T$ - положительное $L_{1}-L_{\infty}$-сжатие на вероятностнол пространстве $(X, \nu)$, то для любой функијии $\varphi \in L_{1}(X, \nu)$ найдется $\bar{\varphi}$ $L_{1}(X, \nu)$, такая, ито $\left(\varphi+T \varphi+\cdots+T^{n-1} \varphi\right) / n \rightarrow \bar{\varphi}$ почти всюду по мер

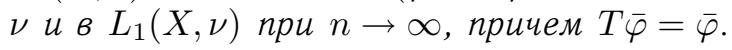


Применяя теорему 3 к оператору $T_{\mu}$ и используя следствие 1 , мы получае лемму 7 .

Лемма 7 доказывает сходимость временны́х средних в теореме 2 .

Сходимость в теореме 3 имеет место также для пространств бесконечной мерь поэтому верна

Лемма 8. Пусть $(X, \nu)$ - пространство с мерой, и пусть $T_{1}, \ldots, T_{m}$ положительные $L_{1}-L_{\infty}$-сжатия. Тогда для любой функции $\varphi \in L_{1}(X, \nu)$ любого $i=1, \ldots, m$ последовательность $c_{n}^{\mu, i}(T) \varphi$ сходится $\nu$-почти всюд прu $n \rightarrow \infty$.

Теперь рассмотрим сжатия в $L_{p}, p>1$.

ЛЕмма 9. Пусть $(X, \nu)$ - пространство Лебега, пусть $p>1$, u пуст $T_{1}, \ldots, T_{m}-$ положительнье $L_{p}$-сжатия.

Для любой функции $\varphi \in L_{p}(X, \nu)$ и для любого $i \in\{1, \ldots, m\}$ последова тельность $\left(1 / p_{i}\right) c_{n}^{\mu, i}(T) \varphi$ сходится $\nu$-почти всюду и в $L_{p}(X, \nu) \kappa$ некоторо функции $\bar{\varphi}_{i} \in L_{p}(X, \nu)$, причем $T_{\mu}\left(\bar{\varphi}_{1}, \ldots, \bar{\varphi}_{m}\right)=\left(\bar{\varphi}_{1}, \ldots, \bar{\varphi}_{m}\right)$.

Это следует из эргодических теорем Акоглу [23] и Лорча [20], примененных оператору $T_{\mu}$.

СледствиЕ 4. В предположениях леммы 9 для любой функции $\varphi \in L_{p}(X, \nu$ последовательность $c_{n}^{\mu}(T) \varphi$ сходится $\nu$-почти всюду и в $L_{p}(X, \nu)$.

Это следствие завершает доказательство сходимости временны́х средних в лем ме 2 .

ЗАмЕЧАНИЕ. Конструкция оператора $T_{\mu}$ может быть слегка обобщенс Пусть, как выше, $L-$ линейное пространство над $\mathbb{R}$ или $\mathbb{C}, T_{1}, \ldots, T_{m}: L \rightarrow$ - линейные операторы и $P$ - стохастическая матрица. Определим операто $T_{P}: L^{m} \rightarrow L^{m}$ формулой

$$
T_{P}\left(v_{1}, \ldots, v_{m}\right)=\left(\tilde{v}_{1}, \ldots, \tilde{v}_{m}\right), \quad \text { где } \tilde{v}_{j}=\sum_{k=1}^{m} p_{j k} T_{j} v_{k} .
$$

Если $P$ имеет стационарное распределение $\left(p_{1}, \ldots, p_{m}\right)$, такое, что все $p_{i}$ стро го положительны, то можно рассмотреть матрицу $P^{*}=\left(p_{i j}^{*}\right)$ заданную форму лой $p_{j k}^{*}=p_{k} p_{k j} / p_{j}$. Если $\mu-$ марковская мера с начальным распределение $\left(p_{1}, \ldots, p_{m}\right)$ и матрицей переходных вероятностей $P$, то из определений ясно, чт $T_{\mu}=T_{P^{*}}$.

\section{§5. Инвариантность предела}

В этом параграфе мы устанавливаем инвариантность пределов и завершае доказательство теоремы 2.

ЛЕмма 10. Пусть $T_{1}, \ldots, T_{m}-$ положительные $L_{1}-L_{\infty}$-сжатия на $L_{1}(X, \nu)$ Пусть $\mu$ - сильно неприводимая $\sigma_{m}$-инвариантная марковская мера на $\Omega_{m}$ Пусть $\varphi_{1}, \ldots, \varphi_{m} \in L_{1}(X, \nu)$ таковьи, ито

$$
T_{\mu}\left(\varphi_{1}, \ldots, \varphi_{m}\right)=\left(\varphi_{1}, \ldots, \varphi_{m}\right) .
$$

Тогда $\varphi_{1}=\cdots=\varphi_{m}=\varphi$ и $T_{i} \varphi=\varphi$ для всех $i=1, \ldots, m$.

Сначала мы устанавливаем аналогичный результат для сжатий в гильбертовы пространствах. 
ЛЕмма 11. Пусть $H-$ гильбертово пространство, а операторь $T_{1}, \ldots$ $T_{m}: H \rightarrow H-$ сжатия. Пусть н есть $\sigma_{m}$-инвариантная марковская меран $\Omega_{m}$. Предположим, что векторы $h_{1}, \ldots, h_{m} \in H$ таковы, что $T_{\mu}\left(h_{1}, \ldots, h_{m}\right)=$ $\left(h_{1}, \ldots, h_{m}\right)$.

Тогда если мера н сильно неприводима, то $h_{1}=\cdots=h_{m}=h u T_{i} h=h n p$ всех $i=1, \ldots, m$.

Основная идея доказательства: если $v_{1}, v_{2}, v_{3}-$ векторы в гильбертовом про странстве, такие, что $\left|v_{1}\right|=\left|v_{2}\right|=\left|v_{3}\right|$ и $v_{1}=\left(v_{2}+v_{3}\right) / 2$, то $v_{1}=v_{2}=v_{3}$. Гиварц в [19] использовал это замечание в доказательстве инвариантности предельно функции в своей эргодической теореме.

ДокАЗАТЕЛЬСтво. Пусть мера $\mu$ имеет начальное распределение $\left(p_{1}, \ldots, p_{m}\right.$ и матрицу переходных вероятностей $P=\left(p_{i j}\right)$. Для $i, j \in\{1, \ldots, m\}$ и $n \in I$ обозначим через $p_{i j}^{(n)}$ вероятность перехода из $i$ в $j$ за $n$ шагов (иначе говоря $\left.p_{i j}^{(n)}=\left(P^{n}\right)_{i j}\right)$.

ПрЕДЛОЖЕНИЕ 1. Пусть $H$ - гильбертово пространство, а $T_{1}, \ldots, T_{m}$ $H \rightarrow H-$ сжатия. Пусть н есть $\sigma_{m}$-инвариантная марковская мера на $\Omega_{m}$ такая, что соответствующая ей цеепь Маркова неприводима. Пусть $h_{1}, \ldots$ $h_{m} \in H$ таковьи, что $T_{\mu}\left(h_{1}, \ldots, h_{m}\right)=\left(h_{1}, \ldots, h_{m}\right)$.

Тогда найдется $r \in \mathbb{R}$, такое, ито $\left|h_{1}\right|=\cdots=\left|h_{m}\right|=r$, причем если $p_{i j}>0$ mo $\left|T_{j} h_{i}\right|=r$.

ДокАЗАТЕЛЬство. Пусть $\left|h_{1}\right| \geqslant\left|h_{i}\right|$ при всех $i=1, \ldots, m$. Так как $h_{1}$ $\sum_{i=1}^{m}\left(p_{i} p_{i 1} / p_{1}\right) T_{1} h_{i}$ и $1=\sum_{i=1}^{m} p_{i} p_{i 1} / p_{1}$, из неравенства треугольника следуе что если $p_{i 1}>0$, то $\left|h_{1}\right|=\left|T_{1} h_{i}\right|=\left|h_{i}\right|$.

Аналогично, $\left|h_{1}\right|=\left|h_{i}\right|$ для всех $i$, таких, что $p_{i 1}^{(2)}>0$, и т. д. Цепь Маркова соответствующая мере $\mu$, неприводима, и мы получаем, что $\left|h_{1}\right|=\cdots=\left|h_{m}\right|$, если $p_{i j}>0$, то $\left|h_{j}\right|=\left|T_{j} h_{i}\right|$.

ПрЕДЛОЖЕНИЕ 2. Пусть $h_{1}, \ldots, h_{n}, h \in H$ maковы, ито $\left|h_{1}\right|=\left|h_{2}\right|=\cdots$ $\left|h_{n}\right|=|h|$. Предположим, что нашлись $\alpha_{1}>0, \ldots, \alpha_{n}>0, \alpha_{1}+\cdots+\alpha_{n}=1$ такие, ито $h=\alpha_{1} h_{1}+\cdots+\alpha_{n} h_{n}$. Тогда $h_{1}=h_{2}=\cdots=h_{n}=h$.

Это прямое следствие условия достижения равенства в неравенстве Коши Буняковского-Шварца.

ПреДЛОЖЕНИЕ 3. Пусть $H-$ гильбертово пространство, $T: H \rightarrow H$ сжатие, а векторы $h_{1}, h_{2} \in H$ таковы, что $\left|h_{1}\right|=\left|h_{2}\right|=\left|T h_{1}\right|=\left|T h_{2}\right|$. Тогд если $T h_{1}=T h_{2}$, mо $h_{1}=h_{2}$.

Если $h_{1} \neq h_{2}$, то $\left|\left(h_{1}+h_{2}\right) / 2\right|<\left|h_{1}\right|$ по предложению 2. Так как $\mid T\left(\left(h_{1}-\right.\right.$ $\left.\left.h_{2}\right) / 2\right)|=| h_{1} \mid$, мы приходим к противоречию с тем, что $T-$ сжатие.

Ниже запись $\left(P P^{T}\right)_{i j}$ означает $(i, j)$-й элемент матрицы $P P^{T}$.

ПреДЛОЖЕНИЕ 4. Пусть $\mu$ есть $\sigma_{m}$-инвариантная марковская мера с непри водимой переходной матрицей $P$. Пусть $T_{\mu}\left(h_{1}, \ldots, h_{m}\right)=\left(h_{1}, \ldots, h_{m}\right)$. Тогд если $\left(P P^{T}\right)_{i j}>0$, mo $h_{i}=h_{j}$.

ДокАЗАТЕЛЬСТВО. По предложению 1 из неприводимости матрицы $P$ следуел что $\left|h_{1}\right|=\cdots=\left|h_{m}\right|$. Заметим, что $\left(P P^{T}\right)_{i j}>0$, если и только если найдется $k$ такое, что $p_{i k}>0, p_{j k}>0$. Так как $h_{k}=\sum_{l=1}^{m}\left(p_{l} p_{l k} / p_{k}\right) T_{k} h_{l}$, из предложения следует, что $h_{k}=T_{k} h_{i}=T_{k} h_{j}$, причем по предложению $1\left|h_{k}\right|=\left|h_{i}\right|=\mid h_{j}$ откуда в силу предложения 3 заключаем, что $h_{i}=h_{j}$, и предложение доказано 
Из предложения 4 следует, что если матрица $P$ неприводима и матрица $P P$ неприводима, то $h_{1}=\cdots=h_{m}$, и лемма 11 доказана.

Из лемм 5 и 11 и следствия 2 вытекает лемма 1.

Лемма 10 получается из леммы 11 приближением интегрируемых функци квадратично интегрируемыми. Пусть $\varepsilon>0$ произвольно. Пусть $\varphi_{1}^{\prime}, \ldots, \varphi_{m}^{\prime}$ $L_{2}(X, \nu)$ таковы, что $\left|\varphi_{i}^{\prime}-\varphi_{i}\right|_{L_{1}}<\varepsilon$. Определим функции $\psi_{1}, \ldots, \psi_{m}$ формулой

$$
\left(\psi_{1}, \ldots, \psi_{m}\right)=\lim _{N \rightarrow \infty} \frac{1}{N} \sum_{n=0}^{N-1} T_{\mu}^{n}\left(\varphi_{1}^{\prime}, \ldots, \varphi_{m}^{\prime}\right)
$$

(предел существует почти всюду и в $L_{1}(X, \nu)$ ). Очевидно, что $\psi_{i} \in L_{2}(X, \nu)$ $\left|\psi_{i}-\varphi_{i}\right|_{L_{1}}<\varepsilon$ для всех $i=1, \ldots, m$ и $T_{\mu}\left(\psi_{1}, \ldots, \psi_{m}\right)=\left(\psi_{1}, \ldots, \psi_{m}\right)$. Операто $T_{\mu}$, будучи сжатием в $L_{1}$ и в $L_{\infty}$, является сжатием и в $L_{2}$ (это следует из тес ремы Рисса о выпуклости или из леммы Лина, см. [21, р. 65]). Теперь, применя лемму 11 , видим, что $\psi_{1}=\cdots=\psi_{m}=\psi$ и $T_{i} \psi=\psi$ для всех $i=1, \ldots, m$ Так как $\left|\psi_{i}-\varphi_{i}\right|_{L_{1}}<\varepsilon$, а $\varepsilon$ произвольно, заключаем, что $\varphi_{1}=\cdots=\varphi_{m}=\varphi$ $T_{i} \varphi=\varphi$ при всех $i=1, \ldots, m$. Лемма 10 доказана.

Лемма 10 и лемма 7 вместе дают теорему 2.

Переходим к доказательству леммы 2.

ЛЕмма 12. Пусть $p>1$, и пусть $T_{1}, \ldots, T_{m}: L_{p}(X, \nu) \rightarrow L_{p}(X, \nu)-$ сжатия Пусть н есть $\sigma_{m}$-инвариантная марковская мера на $\Omega_{m}$. Пусть функци $\varphi_{1}, \ldots, \varphi_{m} \in L_{p}(X, \nu)$ таковы, ито $T_{\mu}\left(\varphi_{1}, \ldots, \varphi_{m}\right)=\left(\varphi_{1}, \ldots, \varphi_{m}\right)$.

Если мера н сильно неприводима, то $\varphi_{1}=\cdots=\varphi_{m}=\varphi$ и $T_{i} \varphi=\varphi$ при все $i=1, \ldots, m$.

Доказательство леммы 12 дословно повторяет доказательство леммы 11 Основное наблюдение состоит в том, что предложение 2 выполнено для про странства $L_{p}(X, \nu)$.

Из лемм 9 и 12 вытекает лемма 2.

Аналог леммы 11 имеет место во всех банаховых пространствах, для которы выполнен аналог предложения 2 (такие банаховы пространства иногда называю строго нормированными). По лемме 6 аналог леммы 1 имеет место в произволь ном рефлексивном строго нормированном банаховом пространстве.

\section{§6. Сильная неприводимость и симметричная сигма-алгебра}

В этом параграфе доказано, что сильная неприводимость неприводимой мар ковской цепи с конечным числом состояний эквивалентна тривиальности ее сим метричной сигма-алгебры.

Пусть $P=\left(p_{i j}\right)-$ стохастическая $m \times m$-матрица. Сопоставим ей неориенти рованный граф $\Gamma_{P}$ с вершинами $1, \ldots, m$, в котором вершины $i_{1}$ и $i_{2}$ соединен ребром, если найдется $j$, такое, что $p_{i_{1} j}>0, p_{i_{2} j}>0$. Иными словами, $\Gamma_{P}$ ест граф инцидентности матрицы $P P^{T}$. (Здесь, как всегда, $P^{T}$ есть транспонирован ная матрица $P$.)

Матрица $P$ сильно неприводима, если и только если она неприводима и гра $\Gamma_{P}$ связен.

Если $\mu-$ марковская мера на $\Omega_{m}$ с матрицей переходных вероятностей $P=$ $\left(p_{i j}\right)$, то вместо $\Gamma_{P}$ будем иногда писать $\Gamma_{\mu}$. 
Напомним, что множество $C \subset \Omega_{m}$ называется симметричным, если для вся кой последовательности $\omega \in C$ все последовательности, полученные из $\omega$ конеч ными перестановками, также лежат в $C$. Сигма-алгебра симметричных событи называется симметричной сигма-алгеброй. Напомним, что сигма-алгебра назь вается тривиальной, если всякое множество, лежащее в ней, имеет меру 0 или 1

ПредлоЖениЕ 5. Неприводимая цепь Маркова с конечным числом состоя ний сильно неприводима, если и только если ее симметричная сигма-алгебр тривиальна.

ДокАЗАТЕЛЬСТВО. Пусть $m-$ число состояний, а $P-$ матрица цепи. Пуст $\alpha_{1}$ - сигма-алгебра, порожденная множествами $\left\{\omega_{1}=i\right\}, i=1, \ldots, m$, а $\alpha_{2}$ сигма-алгебра, порожденная множествами $\left\{\omega_{2}=i\right\}, i=1, \ldots, m$. Григоренко [10 показала, что симметричная сигма-алгебра неприводимой марковской цепи три виальна, если и только если пересечение $\alpha_{1} \cap \alpha_{2}$ тривиально.

Пусть $C \subset \Omega_{m}$ и $C \in \alpha_{1} \cap \alpha_{2}$. Это значит, что найдутся множества $A_{1}, A_{2}$ $\{1, \ldots, m\}$, такие, что $C=\left\{\omega: \omega_{1} \in A_{1}\right\}=\left\{\omega: \omega_{2} \in A_{2}\right\}$.

ПредЛОЖЕНИЕ 6. Если $i$ u $j$ соединень ребром в $\Gamma_{P} u i \in A_{1}$, то $u j \in A_{1}$.

Действительно, если $i$ и $j$ соединены ребром в $\Gamma_{P}$, то найдется $k \in\{1, \ldots, m\}$ такое, что $p_{i k}>0, p_{j k}>0$. Если $i \in A_{1}$, то $k \in A_{2}$, откуда $j \in A_{1}$, и предложени доказано.

Из предложения 6 вытекает, что если граф $\Gamma_{P}$ связен, то сигма-алгебра $\alpha_{1} \cap \alpha$ тривиальна.

ПредЛОЖЕНИЕ 7. Если $A \subset\{1, \ldots, m\}-$-вязная компонента графа $\Gamma_{P}, m$ множество $\left\{\omega: \omega_{1} \in A\right\}$ лежит в сигма-алгебре $\alpha_{1} \cap \alpha_{2}$.

Действительно, определим $B \subset\{1, \ldots, m\}$ формулой $B=\{k:$ существуе $i \in A$, такое, что $\left.p_{i k}>0\right\}$. Если $A-$ связная компонента графа $\Gamma_{P}$, то $\left\{\omega: \omega_{1}\right.$ $A\}=\left\{\omega: \omega_{2} \in B\right\}$, и предложение доказано.

Предложение 7 показывает, что если граф $\Gamma_{P}$ несвязен, то и сигма-алгебр $\alpha_{1} \cap \alpha_{2}$ нетривиальна.

Из предложений 6, 7 следует предложение 5.

Другой способ получить предложение 5 - использовать результат Бежаево и Оселедца из [11], из которого следует, что симметричная сигма-алгебра непри водимой цепи Маркова с конечным числом состояний тривиальна, если и тольк если матрица $P^{T} P$ неприводима. Остается заметить, что неприводимость матри цы $P^{T} P$ равносильна неприводимости матрицы $P P^{T}$.

Гуревич [15] показал в 1968 г., что для конечной неприводимой цепи Мар кова тривиальность симметричной сигма-алгебры равносильна условию полнот циклов Колмогорова, необходимому условию в локальной предельной теорем Колмогорова для цепей Маркова (см. [14]).

\section{§7. Эргодические теоремы и косые произведения}

В этом параграфе доказывается, что средние $c_{n}^{\mu}(T) \varphi$, определенные в $\S 2$, схо дятся для произвольной борелевской вероятностной $\sigma_{m}$-инвариантной меры на $\Omega_{m}$.

ТеоремА 4. Пусть $\mu-$ произвольная $\sigma_{m}$-инвариантная борелевская веро ятностная мера на пространстве $\Omega_{m}$. 
Пусть $(X, \nu)$ - вероятностное пространство, $f_{1}, \ldots, f_{m}: X \rightarrow X-$ ото бражения, сохраняющие меру $\nu, u T_{1}, \ldots, T_{m}-$ соответствующие им опера торы.

Для всякой функции $\varphi \in L_{1}(X, \nu)$ найдется функция $\bar{\varphi} \in L_{1}(X, \nu)$, такая ито $c_{n}^{\mu}(T) \varphi \rightarrow \bar{\varphi}$ в $L_{1}(X, \nu)$ при $n \rightarrow \infty$. Eсли $|\varphi| \log |\varphi| \in L_{1}(X, \nu)$, mo $c_{n}^{\mu}(T) \varphi-$ $\bar{\varphi}$ также и почти всюду.

ДокАЗАТЕЛЬСтво. Рассмотрим отображение $F: \Omega_{m} \times X \rightarrow \Omega_{m} \times X$, определен ное формулой

$$
F(\omega, x)=\left(\sigma_{m} \omega, f_{\omega_{1}} x\right) .
$$

Отображение $F$ есть косое произведение с базой $\left(\Omega_{m}, \sigma_{m}\right)$ и слоем $\left(X, f_{1}, \ldots\right.$ $\left.f_{m}\right)$. Для произвольной борелевской вероятностной $\sigma_{m}$-инвариантной меры $\mu \mathrm{H}$ $\Omega_{m}$ отображение $F$ сохраняет меру $\mu \times \nu$ на пространстве $\Omega_{m} \times X$.

Для всякого $w \in W_{m}, w=w_{1} \ldots w_{n}$, определим отображение $f_{w}: X \rightarrow X$ п формуле

$$
f_{w}=f_{w_{n}} \ldots f_{w_{1}}
$$

Пусть $\varphi \in L_{1}(X, \nu)$. Определим $\widetilde{\varphi} \in L_{1}\left(\Omega_{m} \times X, \mu \times \nu\right)$ по формуле $\widetilde{\varphi}(\omega, x)=$ $\varphi(x)$.

ПрЕДЛОЖЕНИЕ 8. Для почти любого $x \in X$ имеем

$$
\int_{\Omega_{m}} \tilde{\varphi} \circ F^{j}(\omega, x) d \mu(\omega)=\sum_{|w|=j} \mu(w) \varphi \circ T_{w}(x) .
$$

Это предложение по существу принадлежит Какутани [3]; доказательство ег есть прямое вычисление.

Положим

$$
S_{j}^{\mu}(f) \varphi=\sum_{|w|=j} \mu(w) \varphi \circ f_{w} .
$$

Усредним $S_{j}^{\mu}(F)$ по $j=0, \ldots, n-1$, и пусть

$$
C_{n}^{\mu}(f) \varphi=\frac{1}{n} \sum_{j=0}^{n-1} S_{j}^{\mu}(f) \varphi .
$$

Положим $\widetilde{\varphi}_{n}=\frac{1}{n} \sum_{j=0}^{n-1} \widetilde{\varphi} \circ f^{j}$.

Из предложения 8 вытекает

СЛЕДСТвИЕ 5. Для почти любого $x \in X$ имеет место равенство $C_{n}^{\mu}(f)$ $=\int_{\Omega_{m}} \widetilde{\varphi}_{n}(\omega, x) d \mu(\omega)$.

Действительно, $\int_{\Omega_{m}} \widetilde{\varphi}_{n}(\omega, x) d \mu(\omega)=\frac{1}{n} \sum_{j=0}^{n-1} \sum_{|w|=j} \mu(w) \varphi \circ f_{w}(x)=C_{n}^{\mu}(f) \varphi$

СлЕДСТвИЕ 6. Если $\varphi \in L_{1}(X, \nu)$, то последовательность $C_{n}^{\mu}(f) \varphi$ сходитс в $L_{1}(X, \nu)$ при $n \rightarrow \infty$. Если $\varphi$ такова, ито $|\varphi| \log |\varphi| \in L_{1}(X, \nu)$, то последо вательность $C_{n}^{\mu}(f) \varphi$ сходится почти всюду.

Это вытекает из следствия 5, эргодической теоремы Биркгофа-Хинчина дл отображения $F$ и теоремы Винера о мажорируемой сходимости временны́х сред них.

Теперь свяжем средние $C_{n}^{\mu}(f)$, определенные в этом параграфе, и средни $c_{n}^{\mu}(T)$, определенные в $\$ 2$. 
Пусть $w \in W_{m}, w=w_{1} \ldots w_{n}$. Положим $w^{*}=w_{n} \ldots w_{1}$. Заметим, что $T_{w} \varphi=$ $\varphi \circ f_{w^{*}}$. Пусть $\mu$ есть $\sigma_{m}$-инвариантная борелевская вероятностная мера на $\Omega_{m}$ Определим новую меру $\mu^{*}$ на $\Omega_{m}$ формулой $\mu^{*}(w)=\mu\left(w^{*}\right)$. Мера $\mu^{*}$ такж является борелевской, вероятностной и $\sigma_{m}$-инвариантной; ясно, что $\mu^{* *}=\mu$.

ПрЕДЛОЖЕНИЕ 9. $C_{n}^{\mu}(f)=c_{n}^{\mu^{*}}(T)$.

Действительно, формула $T_{w} \varphi=\varphi \circ f_{w^{*}}$ дает

$$
\sum_{|w|=n} \mu(w) T_{w} \varphi=\sum_{|w|=n} \mu(w) \varphi \circ f_{w^{*}}=\sum_{|w|=n} \mu^{*}\left(w^{*}\right) f_{w^{*}}=\sum_{|w|=n} \mu^{*}(w) \varphi \circ f_{w} .
$$

Из предложения 9 и следствия 6 вытекает теорема 4.

\section{§8. Эргодические теоремы для средних, полученных с помощью марковских мер порядка $k$}

В этом параграфе теорема 2 обобщена на суммирование с весами, соответству ющими марковским мерам порядка $k \geqslant 1$.

Борелевскую вероятностную меру $\mu$ на $\Omega_{m}$ будем называть марковской меро порядка $k$, если найдутся неотрицательные величины $p_{i_{1} \ldots i_{k}}$ (начальное распре деление) и $p_{i_{1} \ldots i_{k+1}}$ (вероятности перехода), $i_{1}, \ldots, i_{k+1} \in\{1, \ldots, m\}$, такие, чт для любого $w \in W_{m}, w=w_{1} \ldots w_{n}$, имеем

$$
\mu(w)=p_{w_{1} \ldots w_{k}} p_{w_{1} \ldots w_{k+1}} p_{w_{2} \ldots w_{k+2}} \ldots p_{w_{n-k} \ldots w_{n}} .
$$

При $k=1$ получаем определение обычной марковской меры.

Мера $\mu$, определенная соотношением (14), $\sigma_{m}$-инвариантна, если и тольк если при всех $i_{1}, \ldots, i_{k} \in\{1, \ldots, m\}$ имеет место равенство

$$
p_{i_{1} \ldots i_{k}}=\sum_{i=1}^{m} p_{i i_{1} \ldots i_{k-1}} p_{i i_{1} \ldots i_{k}} .
$$

Всякой марковской мере порядка $k$ на пространстве последовательностей из $r$ символов стандартным образом ставится в соответствие марковская мера порядк 1 на пространстве последовательностей из $m^{k}$ символов. Опишем это соотвел ствие.

Пусть $A=\left\{w \in W_{m}:|w|=k\right\}$. Обозначим через $U$ множество всех конечны слов в алфавите $A$ :

$$
U=\left\{u=u_{1} u_{2} \ldots u_{n}: u_{i} \in A\right\} .
$$

Итак, элементы множества $U-$ это слова, составленные из символов, которы сами являются словами. Для всякого слова $u \in U$ через $u_{1}$ мы обозначае его первый символ, через $u_{2}$ - второй и т.д. При этом $u_{1} \in A \subset W_{m}$, т. $u_{1}=\left(u_{1}\right)_{1} \ldots\left(u_{1}\right)_{k}$, где $\left(u_{1}\right)_{i} \in\{1, \ldots, m\}$.

Нам понадобится также пространство односторонних бесконечных последо вательностей символов алфавита $A$. Обозначим его через $\Omega_{A}$, а сдвиг на не через $\sigma_{A}$.

Теперь пусть $\mu$ - марковская мера на $\Omega_{m}$ с начальным распределением $\left(p_{i_{1} \ldots i_{k}}\right.$ и вероятностями перехода $p_{i_{1} \ldots i_{k+1}}, i_{1}, \ldots, i_{k+1} \in\{1, \ldots, m\}$.

Для любых $w, w^{\prime} \in A$ положим $\tilde{p}_{w}=p_{w_{1} \ldots w_{k}}, \tilde{p}_{w w^{\prime}}=p_{w_{1} \ldots w_{k} w_{k}^{\prime}}$, если $w_{2}=$ $w_{1}^{\prime}, \ldots, w_{k}=w_{k-1}^{\prime}$, и $\tilde{p}_{w w^{\prime}}=0$ в противном случае. Величины $\tilde{p}_{w}$ (начально 
распределение) и $\tilde{p}_{w w^{\prime}}$ (вероятности перехода) задают на $\Omega_{A}$ марковскую меру порядка 1 , причем если мера $\mu \sigma_{m}$-инвариантна, то мера $\tilde{\mu} \sigma_{A}$-инвариантна.

Далее, легко видеть, что граф $\Gamma_{\tilde{\mu}}$, определенный в $\$ 6$, имеет не менее $m^{k-}$ компонент связности (вершины $w$ и $w^{\prime}$ в нем могут быть соединены ребром только если $\left.w_{2}=w_{2}^{\prime}, \ldots, w_{k}=w_{k}^{\prime}\right)$.

ОПРЕДЕЛЕНИЕ 4. Марковскую меру $\mu$ порядка $k \geqslant 1$ назовем сильно неприво димой, если цепь Маркова, отвечающая мере $\tilde{\mu}$, неприводима, а граф $\Gamma_{\tilde{\mu}}$ имее в точности $m^{k-1}$ компонент связности.

При $k=1$ получаем определение из $\$ 6$.

ТЕОремА 5. Пусть н есть $\sigma_{m}$-инвариантная марковская мера порядка ra $\Omega_{m}$.

Пусть $H$ - гильбертово пространство, а линейные операторы $T_{1}, \ldots, T_{m}$ $H \rightarrow H-$ сжатия. Тогда для всякого $h \in H$ найдется вектор $\bar{h}$, такой, ит $c_{n}^{\mu}(T) h \rightarrow \bar{h}$ в $H$.

Если мера н сильно неприводима, то $T_{i} \bar{h}=\bar{h}$ при всех $i=1, \ldots, m$.

ТЕОРемА 6. Пусть $\mu$ есть $\sigma_{m}$-инвариантная марковская мера порядка на $\Omega_{m}$.

Пусть $(X, \nu)$ - вероятностное пространство и $T_{1}, \ldots, T_{m}: L_{1}(X, \nu)$ $L_{1}(X, \nu)$ - положительные $L_{1}-L_{\infty}$-сжатия. Тогда для всякой функции $\varphi$ $L_{1}(X, \nu)$ найдется функция $\bar{\varphi} \in L_{1}(X, \nu)$, такая, что $c_{n}^{\mu}(T) \varphi \rightarrow \bar{\varphi}$ почти всю ду по мере $\nu$ и в $L_{1}(X, \nu)$.

Если мера н сильно неприводима, то $T_{i} \bar{\varphi}=\bar{\varphi}$ при всех $i=1, \ldots, m$.

Eсли операторы $T_{1}, \ldots, T_{m}$ марковские, mо $\int_{X} \varphi d \nu=\int_{X} \bar{\varphi} d \nu$.

Теоремы 5 и 6 были анонсированы мною в [22].

Пусть $L-$ банахово пространство и $T_{1}, \ldots, T_{m}: L \rightarrow L-$ линейные опера торы. Для всякого $w \in A$ положим $\widetilde{T}_{w}=T_{w_{1}}$. Тем самым для всякого $u \in U$ $u=u_{1} \ldots u_{n}$, определен оператор $\widetilde{T}_{u}=\widetilde{T}_{u_{n}} \ldots \widetilde{T}_{u_{1}}$.

ПредЛОЖЕНИЕ 10. Пусть $\mu-$ марковская мера порядка $k$ на $\Omega_{m}, a \tilde{\mu}$ отвечающая ей марковская мера порядка 1 на $\Omega_{A}$. Тогда

$$
\sum_{|w|=n} \mu(w) T_{w}=\sum_{|u|=n} \tilde{\mu}(u) \widetilde{T}_{u}
$$

ДОКАЗАТЕЛЬСТВО. Для $u \in U, u=u_{1} \ldots u_{n}$, введем слова $b(u), e(u) \in W_{m}$ п формулам $b(u)=\left(u_{1}\right)_{1}\left(u_{2}\right)_{1} \ldots\left(u_{n}\right)_{1}, e(u)=\left(u_{n}\right)_{2} \ldots\left(u_{n}\right)_{k}$. Видно, что $|b(u)|=$ $|u|$ и $\widetilde{T}_{u}=T_{b(u)}$ для всех $u \in U$, а если $\tilde{\mu}(u)>0$, то $\tilde{\mu}(u)=\mu(b(u) e(u))$. Поэтом

$$
\sum_{|u|=n} \tilde{\mu}(u) \widetilde{T}_{u}=\sum_{|u|=n} \mu(b(u) e(u)) T_{b(u)}=\sum_{|u|=n} \mu(b(u)) T_{b(u)}=\sum_{|w|=n} \mu(w) T_{w} .
$$

Это предложение показывает, что утверждения о сходимости временны́х средних составленных с помощью марковских мер порядка $k$, следуют из соответствую щих утверждений для марковских мер порядка 1.

В $\S 4$ описано, как операторам $\widetilde{T}_{w}, w \in A$, и марковской мере $\tilde{\mu}$ ставится в соол ветствие оператор $\widetilde{T}_{\tilde{\mu}}: L^{m^{k}} \rightarrow L^{m^{k}}$. Пусть теперь $H-$ гильбертово пространств и $T_{1}, \ldots, T_{m}-$ сжатия. Положим $\mathbf{H}=H^{m^{k}}$. 
Лемма 13. Пусть мера $а$ сильно неприводима. Пусть вектор $\mathbf{h} \in \mathbf{H}, \mathbf{h}$ $\left(h_{i_{1} \ldots i_{k}}\right), i_{1}, \ldots, i_{k} \in\{1, \ldots, m\}$, таков, ито $\widetilde{T}_{\tilde{\mu}} \mathbf{h}=\mathbf{h}$. Тогда найдется векто $h \in H$, удовлетворяющии условию $T_{i} h=h$ при любом $i=1, \ldots, m$, такои uто $h_{i_{1} \ldots i_{k}}=h$ nри всех $i_{1}, \ldots, i_{k} \in\{1, \ldots, m\}$.

ДокАЗАтЕльство. Цепь, отвечающая мере $\tilde{\mu}$, неприводима, и, согласно предло жению 1 , найдется $r \in \mathbb{R}$, такое, что $\left|h_{i_{1} \ldots i_{k}}\right|=r$ при всех $i_{1}, \ldots, i_{k}$. Равенств $\widetilde{T}_{\tilde{\mu}} \mathbf{h}=\mathbf{h}$ означает, что

$$
h_{i_{1} \ldots i_{k}}=\sum_{i=1}^{m} \frac{p_{i i_{1} \ldots i_{k-1}} p_{i i_{1} \ldots i_{k}}}{p_{i_{1} \ldots i_{k}}} T_{i_{k}} h_{i i_{1} \ldots i_{k-1}} .
$$

Отсюда и из леммы 2 видим, что если $p_{i i_{1} \ldots i_{k}}>0$, то

$$
h_{i_{1} \ldots i_{k}}=T_{i_{k}} h_{i i_{1} \ldots i_{k-1}} .
$$

Если $i, j \in\{1, \ldots, m\}$ таковы, что $p_{i i_{1} \ldots i_{k}}>0, p_{j i_{1} \ldots i_{k}}>0$, то по лемме получаем

$$
h_{i i_{1} \ldots i_{k-1}}=h_{j i_{1} \ldots i_{k-1}} .
$$

Из определения сильной неприводимости легко следует

ПреДЛОЖенИЕ 11. Пусть мера $\mu$ сильно неприводима. Тогда для любых $i$, $\in\{1, \ldots, m\}$ найдутся $i_{1}, \ldots, i_{k} \in\{1, \ldots, m\}$, такие, что $p_{i i_{1} \ldots i_{k}}>0, p_{j i_{1} \ldots i_{k}}>0$

Отсюда и из (16) видим, что при всех $i, j, i_{1}, \ldots, i_{k-1} \in\{1, \ldots, m\}$ имеем

$$
h_{i i_{1} \ldots i_{k-1}}=h_{j i_{1} \ldots i_{k-1}} .
$$

Продолжая по индукции и используя равенства $(15),(16)$, получаем, что при все $i_{1}, \ldots, i_{k}, j_{1}, \ldots, j_{k} \in\{1, \ldots, m\}$ выполнено $h_{i_{1} i_{2} \ldots i_{k}}=h_{j_{1} \ldots j_{k}}=h$ и $T_{i} h=h$ пр всех $i=1, \ldots, m$. Лемма доказана.

Из лемм 13, 5, 11 и 2 следует теорема 5.

Перейдем к доказательству теоремы 6 . Итак, пусть $\nu(X)<\infty, T_{1}, \ldots, T_{m}$ $L_{1}(X, \nu) \rightarrow L_{1}(X, \nu)-$ положительные $L_{1}-L_{\infty}$-сжатия, а $\mu$ - марковская мер порядка $k$ на $\Omega_{m}$.

По теореме Данфорда-Шварца и $L_{1}$-эргодической теореме для оператора $\widetilde{T}$ (см. [21, р. 73]), используя предложение 10 , получим следующий результат:

ЛЕмма 14. Для всякой функции $\varphi \in L_{1}(X, \nu)$ найдется функция $\bar{\varphi} \in L_{1}(X, \nu)$ такая, ито $c_{n}^{\mu}(T) \varphi \rightarrow \bar{\varphi}$ почти всюду по мере $\nu$ и в $L_{1}(X, \nu)$.

Теперь, используя теорему 5 , докажем, что если мера $\mu$ сильно неприводима то $\bar{\varphi}=T_{i} \bar{\varphi}$ при всех $i=1, \ldots, m$.

Это получается из леммы 13 с помощью приближения интегрируемых функци квадратично интегрируемыми точно так же, как лемма 10 получается из лемм 11 в $\$ 5$.

Теорема 6 доказана.

\section{§9. Эргодические теоремы для действий строго марковских полугрупп}

В этом параграфе определены строго марковские полугруппы и получены эр годические теоремы для их действий.

Пусть $A$ есть $m \times m$-матрица с неотрицательными элементами. Слово $w \in W_{m}$ $w=w_{1} \ldots w_{n}$, назовем -допустимым, если $A_{w_{1} w_{2}} A_{w_{2} w_{3}} \ldots A_{w_{n-1} w_{n}}>0$. 
Пусть $G$ - конечно порожденная полугруппа, и пусть $S-$ конечный набор е образующих. Для элемента $g \in G$ обозначим через $|g|_{S}$ его длину по отношенин к $S$ (т. е. длину кратчайшей записи элемента $g$ словом в алфавите $S$ ).

ОПредЕЛЕНиЕ 5. Пусть $G$ - полугруппа с набором образующих $S=\left\{g_{1}, \ldots, g_{k}\right.$ Предположим, что найдется натуральное $m \geqslant k$, отображение $\pi:\{1, \ldots, m\}-$ $\left\{g_{1}, \ldots, g_{k}\right\}$ и неприводимая $m \times m$-матрица $A$, такие, что отображение $w$ $\pi\left(w_{1}\right) \ldots \pi\left(w_{n}\right)$ задает сохраняющую длины биекцию между множеством $A$-допу стимых слов, начинающихся одним из символов $1, \ldots, k$, и полугруппой $G$ («со храняющая длины» означает здесь, что элементу $g \in G$ отвечает слово длин $\left.|g|_{S}\right)$. В таком случае будем называть $G$ строго марковской по отношению набору образующих $S$. Матрицу $A$ будем называть матрицей кодирования.

ОПРЕДЕЛЕНИЕ 6. Конечно порожденная полугруппа называется строго марков ской, если у нее есть набор образующих, по отношению к которому она являетс строго марковский.

Очевидные примеры строго марковских групп - свободные группы, свободны произведения и локально свободные группы Вершика (см. [6, 5]).

Определение строго марковских полугрупп более ограничительно, чем опре деление марковских групп Кэннона [17] и Громова [1]. Разумеется, всякая строг марковская группа является марковской, но обратное неверно, как показывае простое

ПрЕдЛОЖЕНИЕ 12. Бесконечная нециклическая строго марковская групп имеет экспоненциильный рост.

Из этого предложения следует, что свободная абелева группа с двумя ил более образующими не является строго марковской.

М. Л. Громов доказал в [1], что все гиперболические группы - марковские.

Вопрос. Верно ли, что все гиперболические группы строго марковские?

Обратное неверно: локально свободные группы Вершика с тремя или боле образующими, очевидно, строго марковские и не гиперболические (см. $[5,6])$.

Интересный частный случай - фундаментальные группы компактных ориен тируемых поверхностей (действия таких групп, как показали Арнольд и Кры лов [9], естественным образом возникают в теории обыкновенных дифференци альных уравнений с комплексным временем).

ГипотЕзА. Фундаментальная группа компактной ориентируемой поверхност рода $g>1$ строго марковская.

Теорема 1 позволяет получить эргодические теоремы для действий строго мар ковских полугрупп.

ТеОрема 7. Пусть $G-$ строго марковская полугруппа по отношению набору образующих $S$. Пусть $G$ действует на вероятностном пространств $(X, \nu)$ сохраняющими меру отображениями, и пусть $T_{g}$ - отображение, со ответствующее элементу $g$.

Тогда для любой функциии $\varphi \in L_{1}(X, \nu)$ найдется функция $\bar{\varphi} \in L_{1}(X, \nu)$ такая, что

$$
\frac{1}{N} \sum_{n=0}^{N-1} \frac{1}{\#\left\{g:|g|_{S}=n\right\}} \sum_{|g|_{S}=n} T_{g} \varphi \rightarrow \bar{\varphi}
$$

почти всюду по мере $\nu$ и в $L_{1}(X, \nu)$ при $N \rightarrow \infty$. 
Если матрица кодирования сильно неприводима, то $T_{g} \bar{\varphi}=\bar{\varphi}$ для всех $g \in G$

Пусть $A$ есть $m \times m$-матрица, а $\pi:\{1, \ldots, m\} \rightarrow S-$ отображение из опреде ления 5 .

Пусть $f_{1}, \ldots, f_{m}$ - отображения, соответствующие элементам $\pi(1), \ldots$ $\pi(m)$. Пусть $\mu-$ мера Парри матрицы $A$. Применяя теорему 1 к отображе ниям $f_{1}, \ldots, f_{m}$ и мере $\mu$, мы получаем теорему 7 . Детали рассуждения буду изложены в моей работе в сборнике памяти Рохлина, который будет опубликова Американским математическим обществом.

\section{ЛИТЕРАТУРА}

1. Gromov M. Hyperbolic groups, In: Essays in Group Theory (Gersten S. M., ed.), M.S.R. Publ., vol. 8, Springer-Verlag, 1987, pp. 75-263.

2. Оселедец В. И. Марковские цепи, косые произведения и эргодические теоремы дл общих динамических систем. Теория вероятностей и ее применения, 10, вып. 3, 551 557 (1965).

3. Kakutani S. Random ergodic theorems and Markoff processes with a stable distribution In: Proc. 2nd Berkeley Symposium Math. Stat. and Prob., 1951, pp. 247-261.

4. Вершик A. М. Динамическая теория роста в группах: энтропия, границы, примерь УМH, 55, вып. 4, 59-128 (2000).

5. Vershik A. M., Nechaev S., Bikbov R. Statistical properties of braid groups in locall free approximation. Preprint IHES/M/99/45, June 1999.

6. Вершик A. М. Численные характеристики групп и соотношения между ними. Записк научных семинаров ПОМИ, 256, 5-18 (1999).

7. Григорчук Р. И. Индивидуальная эргодическая теорема для действий свободны групп. В кн.: Тезисы Тамбовской школы по теории функций, 1986.

8. Григорчук Р. И. Эргодические теоремы для действий свободной группы и свободно полугруппы. Матем. заметки, 65, вып. 5, 779-782 (1999).

9. Арнольд В. И., Крылов А. Л. Равномерное распределение точек на сфере и некоторь эргодические свойства решений линейных обыкновенных дифференциальных уравнє ний в комплексной области. ДАН СССР, 148, № 1, 9-12 (1963).

10. Григоренко Л. А. О $\sigma$-алгебре симметричных событий для счетной цепи Марков Теория вероятностей и ее применения, 24, №1, 198-204 (1979).

11. Бежаева 3. И., Оселедец В. И. О симметричной сигма-алгебре стационарного мар ковского хариссовского процесса. Теория вероятностей и ее применения, 41, вып. 869-877 (1996).

12. Dunford N., Schwartz J. T. Convergence almost everywhere of operator averages J. Rational Mech. Anal., 5, 129-178 (1956).

13. Hopf E. The general temporarily discrete Markoff process. J. Rational Mech. Anal., 3 13-45 (1954).

14. Колмогоров $A$. H. Локальная предельная теорема для классических цепей Марков Изв. АН СССР, 13, № 4, 281-300 (1949).

15. Гуревич Б. М. Локальная предельная теорема для цепей Маркова и условия тип регулярности. Теория вероятностей и ее применения, 13, вып. 1, 183-190 (1968).

16. Nevo $A$. Harmonic analysis and pointwise ergodic theorems for non-commutin transformations. J. Amer. Math. Soc., 7, 875-902 (1994).

17. Cannon J. The combinatorial structure of cocompact discrete hyperbolic groups. Geon Dedicata, 16, 123-148 (1984).

18. Nevo A., Stein E. M. A generalization of Birkhoff's pointwise ergodic theorem. Act Math., 173, 135-154 (1994).

19. Guivarc'h Y. Généralisation d'un théorème de von Neumann. C. R. Acad. Sci Pari 268, 1020-1023 (1969). 
20. Lorch E. R. Means of iterated transformations in reflexive vector spaces. Bull. Ame Math. Soc., 45, 945-947 (1939).

21. Krengel U. Ergodic Theorems. Walter de Gruyter, Berlin-New York, 1985.

22. Буфетов А. И. Эргодические теоремы для нескольких отображений. УМН, 54 вып. 4, 159-160 (1999).

23. Akcoglu M. A pointwise ergodic theorem in $L_{p}$-spaces. Canad. J. Math., 27, 1075-108 (1975).

Независимый московский университет 